For Internal Distribution Only

Accelerator Division

Alternating Gradient Synchrotron Department

BROOKHAVEN NATIONAL LABORATORY

Upton, New York 11973

Accelerator Division

Technical Note

AGS/AD/Tech. Note No. 432

Some Views on the Structure Layout of the

A5 Kicker Power Supply Discharging Housing

Jian-Lin Mi

April 3, 1996 
$+2$

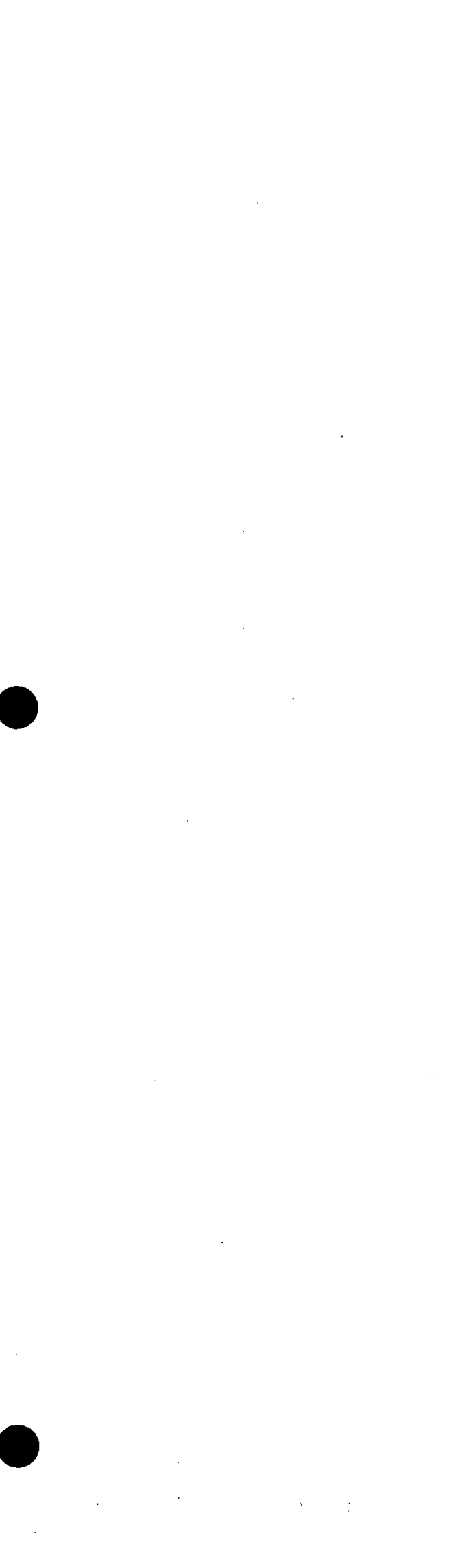

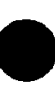

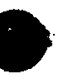

○

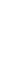

政

,
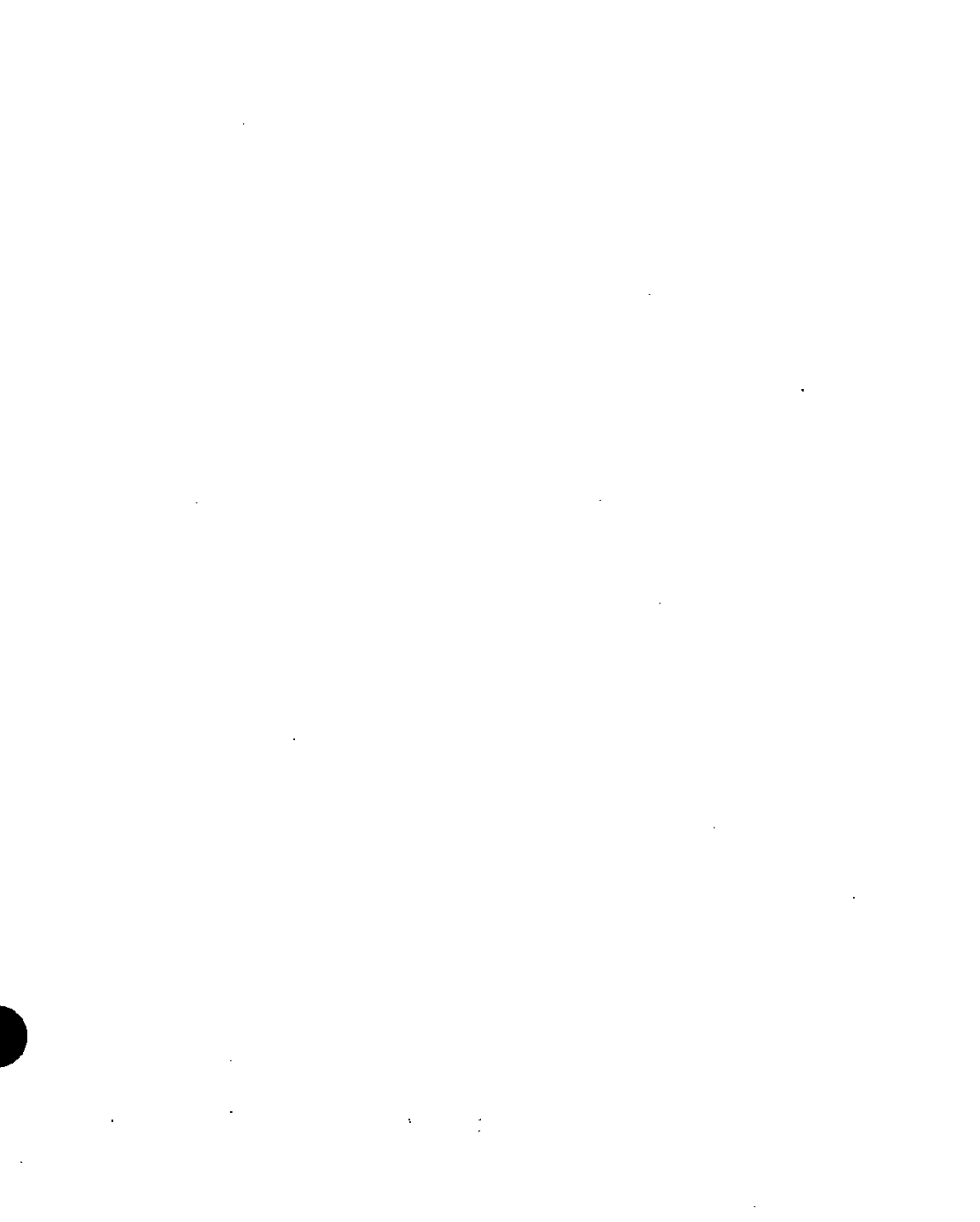


\title{
Some Views On The Structure Layout \\ Of The A5 Kicker Power Supply Discharging Housing
}

\author{
Jianlin Mi \\ Accelerator Division, AGS Department \\ Brookhaven National Laboratory, Upton, Long Island, NY 11973
}

\subsection{INTRODUCTION:}

In order to accommodate a variety of injection schemes into the AGS from the Booster, the AGS A5 fast kicker pulser has to be re-designed. The new upgraded system will be based on a pulse forming line (PFL). Therefore, at each end of the PFL cables, terminations and a switch tube will be required.

There are three schemes for the A5 discharging housing mechanical structure design. All of them have a coaxial structure design in order to reduce the discharging loop inductance. But, depending on the cable feed-through and the connection to the kicker magnet, there are three different layouts. Each design has its advantages and weaknesses. We should weigh these advantages and disadvantages.

\subsection{DISCUSSION:}

This section will describe each of the three designs and will discuss their advantages and disadvantages. Each of the designs has the capability for terminating up to three $50 \mathrm{Ohm}$ coaxial cables. They also contain a switch tube and a matching resistor. They will be filled with an insulating or cooling fluid such as Fluorinert.

\subsection{Layout 1:}

This design is shown in figure 1. The coaxial structure is horizontal. 


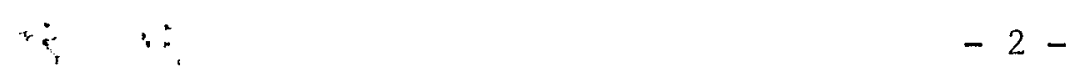

\section{Advantages:}

This layout is designed in coaxial structure which includes the matching discharge resistor and the switch, a CX1 168 thyratron. This design has an advantage of transmission of the pulse waveform through the thyratron and the resistor without or less waveform distortion and with a fast frequency response. The coaxial structure design can reduce the noise leaking out of the housing, which can affect itself and other nearby instruments.

\section{Disadvantages:}

Because the thyratron discharging the high energy stored in the PFL ( or energy stored in the cable ) results in a fast high current pulse, it will create a very strong noise source that can disturb the power supply control and the other instruments in a good operation. This noise problem is the one of the main questions in high power modulator operation.

In this arrangement, the matching resistor is in the lower voltage part of the layout, but the thyratron cathode is located at the high voltage extremity. So the heater, reservoir, bias, prime power supply and trigger source must be floated at the operating high voltage potential. A high voltage isolation transformer has to be used to feed the $\mathrm{AC}$ power to all of the auxiliary power supplies and the noise will leak out through the wire to the filament, reservoir and bias. In order to contain the noise, a large housing is needed for the system, since the high voltage isolation transformer is not a small one, and it thus becomes a good antenna to transmit the noise out.

\subsection{Layout 2:}

This scheme is shown in figure 2.

\section{Advantages:}

This layout also has a good coaxial structure containing the thyratron and the matching resistor which feed the pulse power of the power supply into the kicker magnet. This scheme is very good since it reduces the load inductance which reduces the energy stored in the inductor and gives a fast the rise time. It also reduces the waveform distortion while transmitting the pulse wave. This can reduce the noise leaking out of the housing and improve the other instrument operation around the kicker vicinity.

\section{Weaknesses:}

Because the layout is located in horizontal plane, the transformer oil or Flurinert will not flow or move easily by convection. 
Due to the heating of the thyratron, bubbles will be created and the bubbles will move to the upper wall of the cylinder. These bubbles are not very good. Because these bubbles' electrical constant is less than the cooling liquid electrical constant, the electrical field intensity will double or more. So the bubble will electrically break down and the operation of the thyratron will deteriorate.

Because of the corona and discharging at sharp edge locations, the cooling liquid, transformer oil or fluorinert will carbonize. The carbonized dust will move around in the liquid and some of the dust will deposit on the upper side of the thyratron ceramic surface. Since the machine runs for long periods of time, the dust will damage the thyratron due to arcing on its ceramic surface.

The cable can't be fed through straight because there is not enough room and will protrude into the AGS aisle. So the cable must be fed through at a right angle. The right angle connection has almost the same inductance as the loop created in connecting the feed through to the housing in figure 3.

\subsection{Layout 3:}

Taking into account the disadvantages and advantages above, the layout in figure 3 is developed. The advantages and disadvantages are as follows:

\section{Advantages:}

The coaxial construction in cable connection, matching resistor and thyratron switch has a good waveform transmission with a low waveform distortion and has good noise shielding.

The vertical location position of the coaxial housing is good for the transformer oil or Fluorinert circulation or convection. In this arrangement the thyratron cooling is more efficient, and prevent the coolant from becoming too hot.

If bubbles are created for any reason, they will float to the top of the cylinder where the electric field strength is much lower than the area near the resistor and the thyratron. In other words, it reduces the possibility of arcing and corona. Because the cylinder is vertical, the arcing dust will sink to the bottom where the high voltage condition is not as critical as at the high voltage end and the pulse width of the amplitude voltage is only $0.14 \mu \mathrm{s}$. In addition, the voltage is not as high as it is near the anode of the thyratron.

The thyratron cathode is at the terminal of the kicker magnet. It is thus easier to isolate the pulse high voltage than it is at the cable end. An additional advantage with this arrangement is that some room is available for doing some adjustment for developing or improving the load 
pulse current waveform.

The cable is connected to the matching resistor directly. That reduces the inductance of this connection and compromises the increased loop inductance at the feed through connection.

\section{Disadvantage:}

Because the connection between the thyratron cathode and the kicker feed-through is at right angle, it results in a higher inductance. This inductance when compared to the amount of the whole coaxial structure will slightly affect the rise time of the pulse front edge and the waveform clear. It is necessary to reduce the inductance in this place to as low a value as possible.

\subsection{CONCLUSION:}

Due to the major advantages given in section 2.3 , it is recommend that the scheme of figure 3 is employed in the upgraded pulsing of the A5 kicker.

During the consideration of these layouts, discussions were held with many people which resulted in many useful ideas. Please let me thank Andy Soukas, Chris Pappas, Andy McNerney, Arling Zhang, Joe Tuozzolo, and others who gave me very sincere and useful advice. 


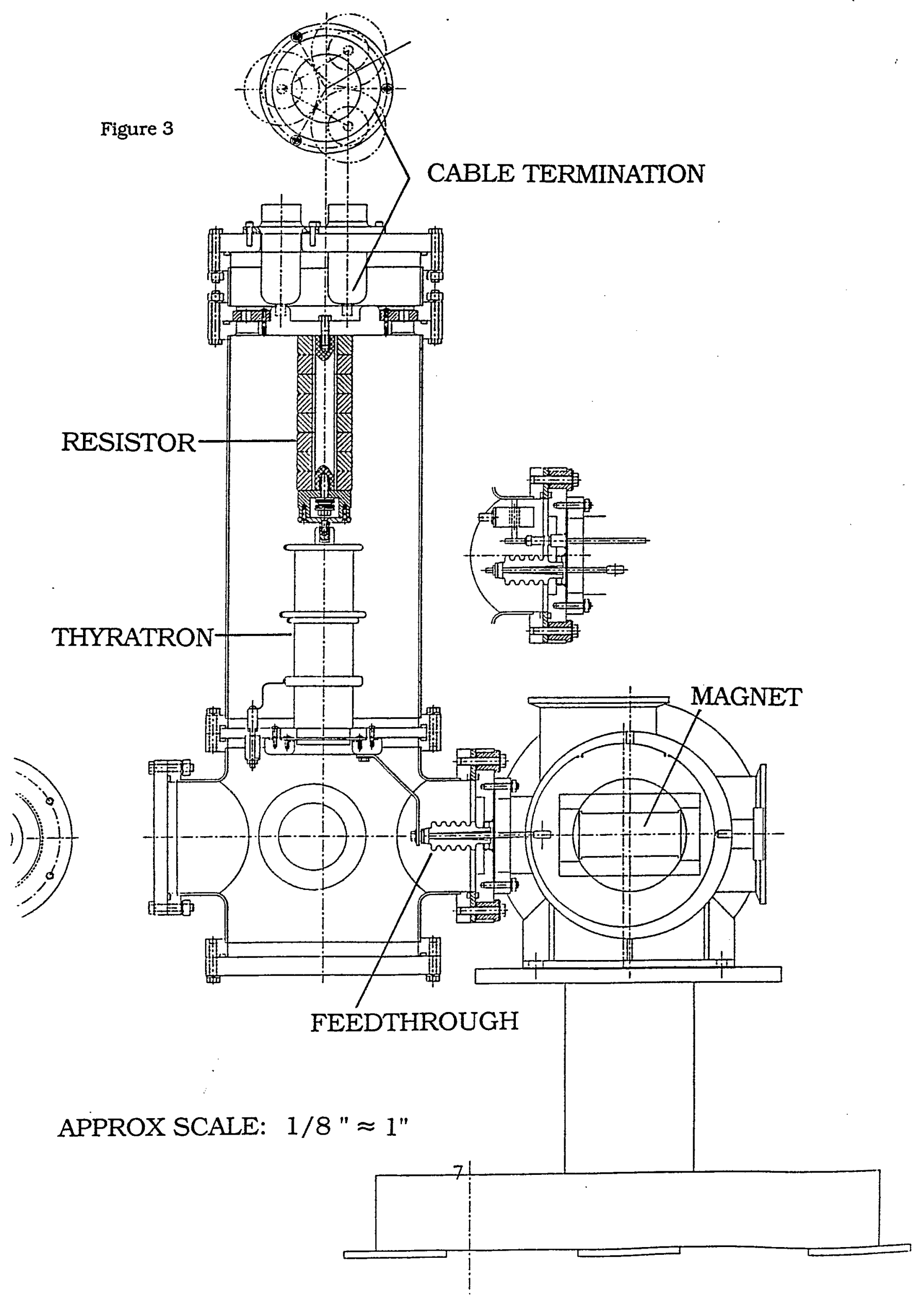




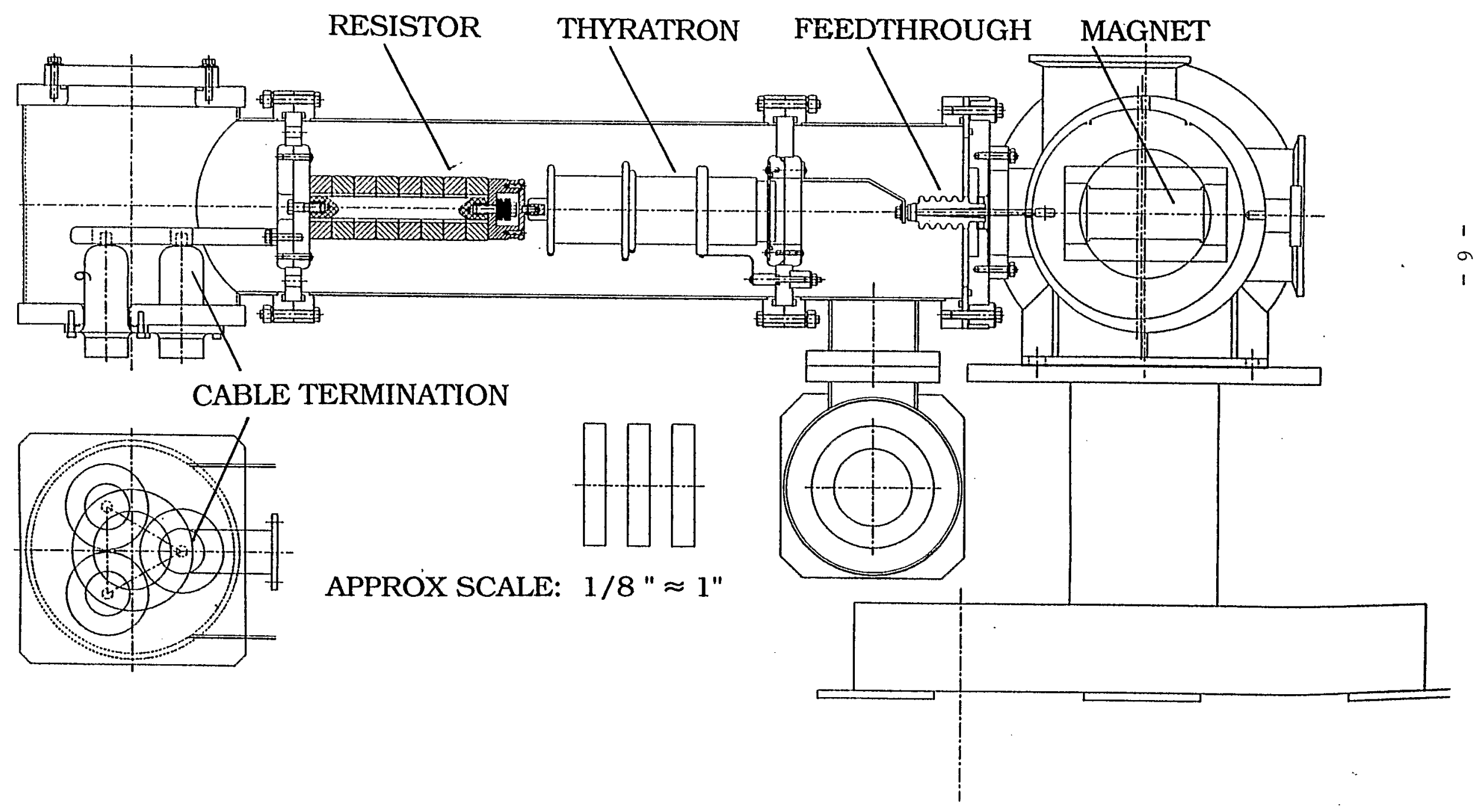



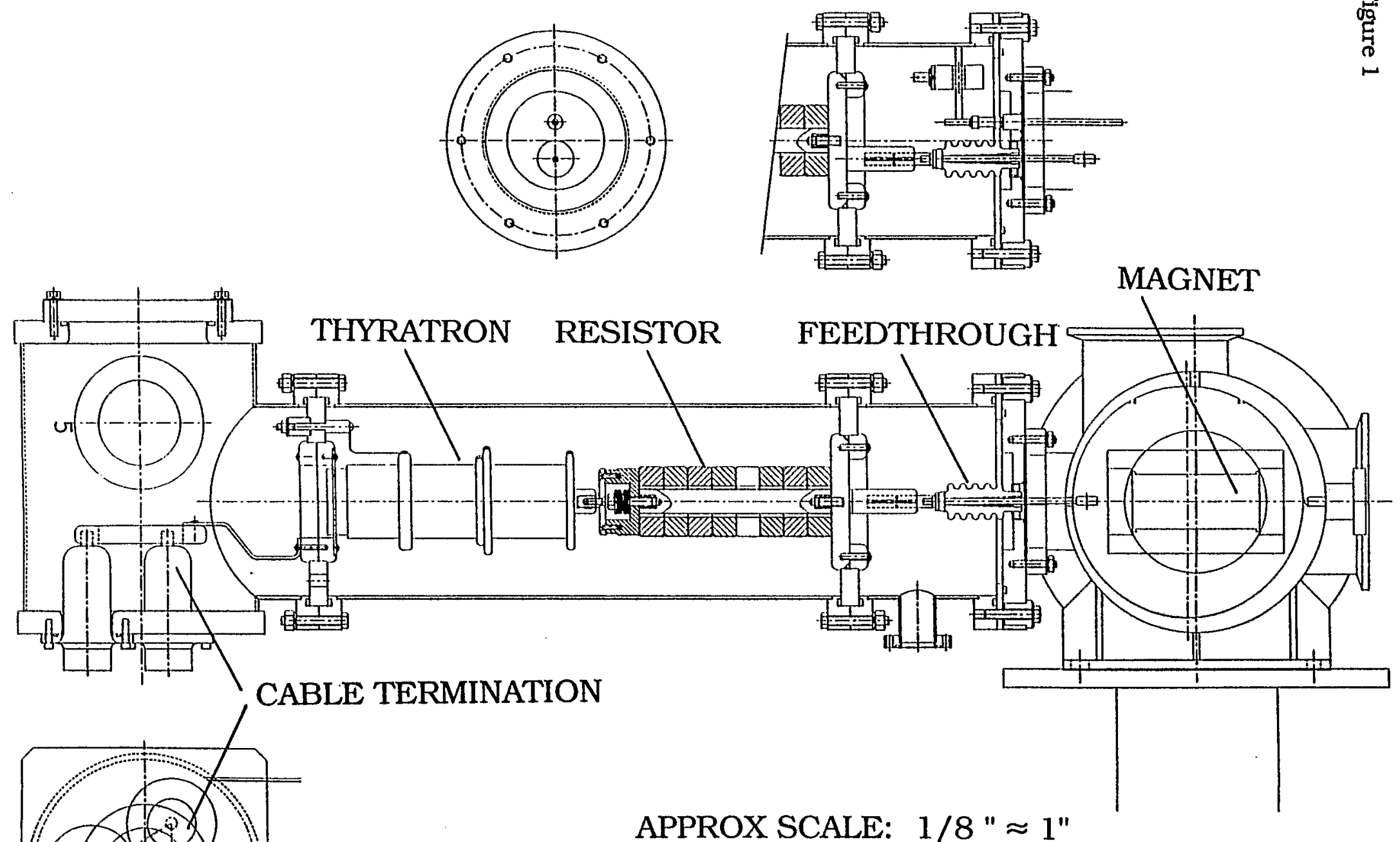

APPROX SCALE: $1 / 8 " \approx 1 "$ 